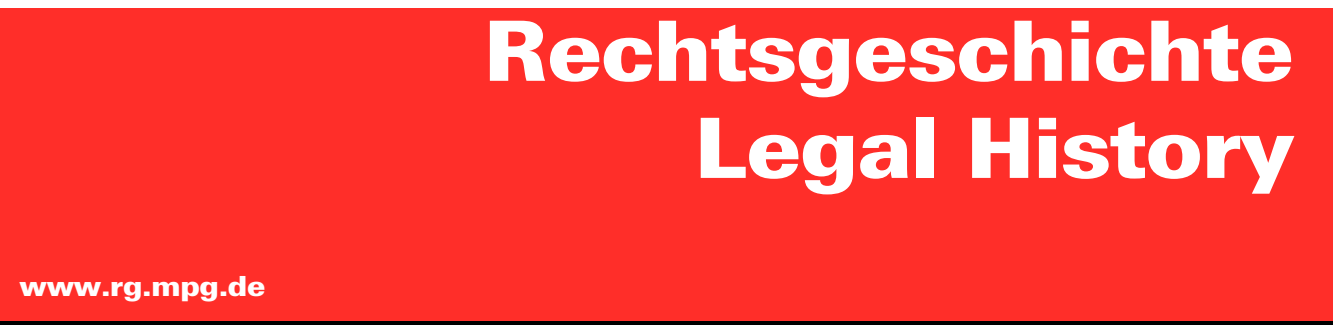

http://www.rg-rechtsgeschichte.de/rg26

Zitiervorschlag: Rechtsgeschichte - Legal History Rg 26 (2018)

$\operatorname{Rg} 26_{2018}$

$438-440$

http://dx.doi.org/10.12946/rg26/438-440

\title{
Karl-Heinz Lingens*
}

\section{Ausgehandelte Ehre}

[Negotiating Noble Titles]

\footnotetext{
* Max-Planck-Institut für europäische Rechtsgeschichte, Frankfurt am Main, lingens@rg.mpg.de
}

Dieser Beitrag steht unter einer Creative Commons cc-by-nc-nd 3.0 
Ausbildung in Deutschland oder an der estnischen Universität in Dorpat (Tartu) trugen dazu bei. Interessant, weil der großen europäischen Erzählung der langsamen Durchsetzung des inquisitorischen gegenüber dem akkusatorischen Verfahren widersprechend, sind die Befunde zum Strafprozessrecht. Im Zivilprozess dagegen findet ein Verrechtlichungs- und Professionalisierungsprozess statt, der sich in das vom westlichen Europa geprägte Bild einfügt. Was die Rechtsquellen angeht, so geben die Analysen, die insbesondere Peter Oestmann für das Reichskammergericht durchgeführt hat, auch einen Schlüssel zum Verständnis für diesen Fall: Die Statutentheorie konnte manches im Nachhinein rechtfertigen, eine wirklich handlungsleitende normative Kraft hatte sie nicht. Man wusste, was recht war.

$\mathrm{Zu}$ welchem Schluss kommt die Studie? Selbst der Staat der Moderne, so beginnt Pihlajamäki seinen Rückblick, kämpfte in seinem Versuch um

\section{Karl-Heinz Lingens Ausgehandelte Ehre*}

Regina Dausers an der Schnittstelle zweier Normensysteme angesiedelte Augsburger geschichtswissenschaftliche Habilitationsschrift ist eine äußerst ergiebige Fundgrube für Rechtshistorikerinnen und Rechtshistoriker, zu deren Interessengebiet Fragen der Multinormativität gehören. Der Fokus der Autorin ist auf die königliche Ehre im Jahrhundert nach dem Westfälischen Frieden gerichtet, wie sie von einem Herrscher beansprucht und von anderen zugestanden oder verweigert wurde. Sichtbaren Niederschlag findet dieser Konkurrenzkampf vor allem in den von der Autorin sowohl quantitativ wie qualitativ ausgewerteten Titulaturen der »völkerrechtlichen« Verträge, welche schon nach zeitgenössischer Auffassung das positive Völkerrecht spiegelten. Diesen praxisorien-
Rechtsvereinheitlichung oder imperiale Ausdehnung mit Pfadabhängigkeiten, Praktiken, Privilegien und Traditionen. Frühneuzeitliche zusammengesetzte Staatlichkeit wie die des schwedischen Imperiums scheiterte bei ihren Bemühungen. Zu stark waren nicht zuletzt die Prägungen durch die deutschen Länder und das gemeine Recht, zu stark auch die überkommene Gerichtsverfassung und ihre Institutionen. Trotz achtzigjähriger Dauer konnte die schwedische Herrschaft die Rechtsgeschichte Livlands nicht dauerhaft prägen - ein aus in verschiedene nationale Archive verstreuten Quellen destillierter Befund, der die vielsprachige und von nationalen Erzählungen geprägte Forschungstradition seit dem 19. Jahrhundert aufnimmt, um wichtige Archivstudien ergänzt und eindrucksvoll in rechtshistorisch-vergleichende analytische Kategorien von heute übersetzt.

tierten Normbereich zieht Dauser ganz bewusst als primäre Quellengrundlage heran, da dessen enorme Publizität, wie sie an mehreren Beispielen nachweisen kann, unbestritten und für die Akteure erkennbar handlungsleitend war.

Bevor sich Dauser mit der Analyse von 453 Dokumenten (hauptsächlich verschiedene Arten von Verträgen, aber auch Ratifikationen: Verzeichnis 326-353) den »quantifizierenden Perspektiven« (105-154) widmet, lässt sie nach einer sachlichen und methodischen Einführung (11-38) die zeitgenössische Literatur zum Titulaturgebrauch zu Wort kommen (39-104). Die ausgewählten Traktate von John Selden, Abraham de Wicquefort, Zacharias Zwanzig, Johann Christian Lünig und Jean Rousset de Missy decken das zeitliche Spek-

* Regina Dauser, Ehren-Namen.

Herrschertitulaturen im völkerrecht-

lichen Vertrag 1648-1748 (Norm und

Struktur 46), Köln, Weimar, Wien

2017, 355 S., ISBN 978-3-412-50590-5 
trum aus unterschiedlicher und kritischer Perspektive (Rechtsgeschichte, Zeremonialwissenschaft, diplomatische Ausbildung und Praxis) ab. Eine gerade auch für die Völkerrechtsgeschichte zentrale Erkenntnis der Studie, »dass die herkömmliche Vorstellung von einer Rangfolge, im ius praecedentiae formuliert und verteidigt, nicht in einer raschen >Erfolgsgeschichte vom Gleichheitskonzept abgelöst wurde«, wird hier schon deutlich. Zwar propagieren die drei letztgenannten Autoren im 18. Jahrhundert - nicht zuletzt im Interesse ihrer Landesherren - das Leitbild souveräner Gleichheit und daraus resultierender Gleichrangigkeit, doch in der Präsentation ihrer Beispiele greifen sie immer wieder auf das tradierte Schema der Hierarchie zurück.

Titulaturen waren im Untersuchungszeitraum veränderliche, sehr flexibel einsetzbare und eingesetzte Instrumente zur Formulierung konkreter Macht- und Geltungsansprüche. Ausdruck eines Strebens nach Höherem waren der ursprünglich dem Kaiser vorbehaltene Majestas-Titel und Attribute wie Potentissimus, deren Verwendung Dauser im zweiten Teil ihrer Arbeit quantifizierend nachgeht (105-154). Während die »Aufsteiger« im zähem Ringen der (Bündnis-)Vertragsverhandlungen darauf abzielten, solche königlichen Ehren nach und nach zu ergattern und sich so unter den gekrönten Souveränen zu etablieren, versuchten die von diesem Aufstieg bedrohten Mächte ihrerseits - dem Kaiser kommt in dieser Gruppe eine Sonderrolle zu - solche Ansprüche abzuwehren, solange sie nicht die Hilfe des Vertragspartners dringend benötigten oder ihm sonst eine Gunst erweisen wollten. Titulaturen waren insofern ein Handelsgegenstand oder, wie Dauser es ausdrückt, Teil eines >Belohnungssystems` (154). Für diese Erkenntnisse ausgewertet wurden Verträge der Könige Frankreichs, Savoyen-Sardiniens und des Zaren sowie von Portugal, England / Großbritannien, Brandenburg-Preußen und des österreichischen Zweiges der Habsburger, deren aus unterschiedlichen Gründen wechselnder Titulaturgebrauch in den Fallbeispielen des umfangreichen abschließenden Teils (155-299) auf einer erweiterten Quellenbasis vertiefend thematisiert wird.

Diese vier Detailstudien stellen, auch unter rechtsgeschichtlichem Aspekt, das Kernstück der Arbeit dar. Die überzeugenden Ergebnisse eröffnen auch Nachbarwissenschaften neue Fragestellungen und geben Anstöße zu weiteren Forschungen. Deutlich herausgearbeitet werden das bis in die
Einzelheiten gehende Ineinandergreifen und vielfältige Überschneidungen der Normbereiche Ehre und Recht. In »Portugal um 1648: Johann IV. von Braganza und der Streit um den wahren Rex Lusitaniae« geht es um die Versuche des neuen portugiesischen Königs, gegen den Widerstand der spanischen Rivalen in Münster und Osnabrück seine Stellung im europäischen Rahmen festlegen $\mathrm{zu}$ lassen. Angesichts der Nichtzulassung seiner Gesandten gelang dies nur ansatzweise, die Schweden konnten zumindest die Aufnahme von Rex et Regnum Lusitaniae (die Formel ist auch für die Bewertung verfassungshistorischer Diskussionen im Reich von Bedeutung) in das Instrumentum Pacis Osnabrugense durchsetzen. Damit wurde völkerrechtlich als Teilerfolg eine begrenzte Anerkennung erreicht, während die für die Ehre wichtigen Präzedenzfragen - Johann IV. hatte seinen Gesandten hier an früheren päpstlichen Rangordnungen orientierte Instruktionen mitgegeben - gar nicht in Angriff genommen werden konnten. Diese standen im zweiten Fallbeispiel »England nach der Glorious Revolution: Wilhelm III. von Oranien - ein >besserer Stuart ?? von Anfang an im Mittelpunkt. Ausgehend von den einschlägigen Erfahrungen des neuen Herrschers als niederländischer Generalstatthalter schildert Dauser detailliert die Auseinandersetzungen um die Prädikate bei der Titulierung, die in zahlreichen Varianten ihren Niederschlag fand. Traditionelle rangrechtliche Argumentationslinien bestimmten weiterhin Denken und Handeln von Kaiser und Königen: Wenn sich Wilhelm auf die Gleichheit der Könige berief, »bezog [er] sich bei näherem Hinsehen nur auf die Gleichheit mit den besonders bevorzugten Monarchen « (219). Ursprünglich rechtliche Argumentationen ließen sich also auch für andere Ziele instrumentalisieren. Für Rechtshistoriker ebenso interessant ist Dausers Beobachtung, dass ehrenhafte Titulaturen oft zunächst in Handschreiben von Herrschern auftraten, bevor sie in einer späteren Phase der bilateralen Beziehungen Eingang in die förmlichen Verträge fanden. Auch hier gibt es Parallelen zum Rechtsbereich: Das spanische Königspaar akzeptierte in den Auseinandersetzungen nach dem Frieden von Utrecht einen Brief Georgs I. von England mit dem Versprechen der Rückgabe Gibraltars, da ein inhaltsgleicher förmlicher Vertrag politisch nicht durchsetzbar war.

Wie sich Ehren-Titel und Territorien des Herrschers zueinander verhielten, verfolgt Dauser in ihrem dritten Beispiel »Preußen 1700-1732: der 
preußische Königstitel, eine Titulatur saus dem Setzkasten «. Auch wenn ein souveräner Herrscher theoretisch sich seinen Titel selbst gab, war es doch ein Gebot politischer Klugheit, den Gebrauch im Vorfeld abzusichern. König >von Preußen< oder >in Preußen bedeutsamer Unterschied, ein Jurist hätte dies mit Blick auf die aus dem Titel abzuleitenden Rechtsansprüche (aktuelles Beispiel Mazedonien) wohl noch stärker betont. Sehr aufschlussreich sind auf der anderen Seite die Beobachtungen der Autorin zum Namen »Oranien« als »freischwebendes Etikett«, das von den Nachkommen des Hauses unabhängig von dem namengebenden Territorium mit anderem Besitz verbunden werden konnte (254). Maßgebende Instanz für derartige Fragen war traditionell der Kaiser, dessen entscheidende Stellung allerdings zunehmend angefochten wurde. Kompliziert wurde es, als nach 1745 im habsburgischen Haus Erbe und Kaisertitel verschiedenen Personen zugeordnet waren: "Maria Theresia und der Kaiserinnentitel - eine sausgeliehener Würde?« ist das letzte Fallbeispiel des Buches über- schrieben, in dem es um die problembehafteten Versuche Wiens geht, angesichts der königlichen Machtstellung und kaiserlichen Würde der Monarchin an den kaiserlichen Vorrechten festzuhalten. Wie bei den anderen Kapitelüberschriften kommt auch hier die Fähigkeit der Autorin zur knappen und präzisen Formulierung zum Ausdruck, welche die Lektüre des Buches insgesamt sehr angenehm macht.

Die an der Person orientierte Normenordnung der Ehre und das stärker zu Institutionalisierung tendierende System des Rechts gemeinsam zu betrachten ist, wie die vorliegende Arbeit eindrucksvoll bestätigt, vielversprechend. Fragen nach Normerzeugern und `Richtern`, nach Präjudizien und Protesten, Umgehungs- und Streitvermeidungsstrategien, bi- oder multilateralen Lösungen, Ausschluss und Zurücksetzung und viele andere mehr verdienen stärkere Beachtung. Regina Dausers Methode und Ergebnisse sind dabei richtungsweisend.

\section{Elisabetta Fiocchi Malaspina \\ Ius gentium europaeum e la sua prassi: a proposito delle raccolte di documenti diplomatici*}

Tre sono i codici al centro del volume di Massimo Panebianco: il Codex juris gentium diplomaticus redatto dal matematico, filosofo, diplomatico e giurista Gottfried Wilhelm von Leibniz nel 1693; il Codex Italiae diplomaticus di Johann Christian Lünig, pubblicato in quattro tomi nel decennio compreso tra il 1725 e il 1735 a Francoforte e a Lipsia e il Corps universel diplomatique $d u$ droit des gens di Jean Dumont edito ad Amsterdam tra il 1726 e il 1731 .

\begin{abstract}
Il discorso diplomatico si snoda tra l'analisi del contesto storico e sociale di una Europa in continua trasformazione e l'evoluzione del diritto delle genti: quest'ultimo viene dipinto attraverso la nascita dei codici diplomatici, "collocato « non solo nella realtà italiana, ma in quella europea e successivamente universale, con particolare attenzione agli Stati italiani preunitari, all'Europa pre e post Westfalia e ricondotto alle sue radici dall'età antica sino a quella moderna.
\end{abstract}

\footnotetext{
* Massimo Panebianco, Introduzione alla codicistica del Jus gentium europaeum. Codice Lünig-Leibniz-Dumont, Napoli: Editoriale Scientifica 2016, 272 p., ISBN 978-88-6342-936-7
} 